\title{
Passive control around the two-dimensional square back Ahmed body using porous devices
}

\author{
Charles-Henri Bruneau (*), Patrick Gilliéron $\left(^{* *}\right)$ and Iraj Mortazavi $\left(^{*}\right)$ \\ (*) Mathématiques Appliquées de Bordeaux \\ CNRS UMR 5466, INRIA FUTURS Equipe MC2 \\ Université Bordeaux 1, 351 cours de la Libération, F-33405 Talence \\ bruneau@math.u-bordeaux1.fr,mortaz@math.u-bordeaux1.fr \\ (**) Technocentre Renault, Direction de la Recherche, \\ Groupe Mécanique des fluides et aérodynamique \\ patrick.gillieron@renault.com
}

\begin{abstract}
The control of two-dimensional flows around the square back Ahmed body is achieved using porous devices added on some parts of the body. The square back Ahmed body is considered either in an open domain or on top of a road. The modelling of the flow in different media is performed by means of the penalisation method. A good choice of the location of the porous interfaces yields a significant improvement of the aerodynamic quantities, specially for the square back body.
\end{abstract}

\section{Introduction}

The research developed today in car aerodynamics is carried out from the point of view of the durable development. Some car companies as Renault have the objective to develop control solutions able to reduce at least $30 \%$ of the aerodynamic drag of the vehicles without constraints on the design, the comfort, the storage or the safety of the passengers [16]. Thus, it is necessary to modify locally the flow, to remove or delay the separation position or to reduce the development of the recirculation zone at the back and of the separated swirling structures [21]. This can be mainly obtained by controlling the flow near the wall with or without additional energy using active or passive devices $[17,18]$. The use of passive systems of control remains however always privileged for obvious reasons of cost. Significant results can be obtained using simple techniques [30]. In practise, the flow control is obtained when the wall pressure distribution is successfully modified on the back and on the rear window, using various adapted devices which change locally the geometry. Control experiments in wind tunnel on reduced or real ground vehicles are performed [28] and measurements of the wall static pressures and of the aerodynamic torque [29] allow to quantify the effect of the control [20]. However, due to the design constraints, the real gain is rather weak and so new control techniques have to be developed.

Among these new techniques in progress, separate devices located in front of or behind the vehicle can be used to reduce the development of the recirculation zone on the rear window or at the back and the interactions of the swirling wake structures. Reductions of the aerodynamic drag up to $97 \%$ are measured on the front part of a circular cylinder in [26]. Interesting results are also obtained with devices located stream wise or transverse 
to the upstream flow behind the back [19]. Another similar approach is to put devices along the wall to generate vortical structures in order to shift the separation line and to reduce the aerodynamic drag. The swirling structures increase the local turbulence of the flow and the energy transfers in the boundary layer. Therefore the velocity increases near the wall. A drag reduction close to $40 \%$ is obtained on wing profiles in [5]. The extension of this technique to more complex geometries should be possible and could defer separation lines towards the back. Furthermore, significant reductions of drag up to $50 \%$ are obtained using artificially rough surfaces [32]. Applied in the areas of weak curvature radius, this technique could decrease the aerodynamic drag.

Self-adapting surfaces made of special coatings can also be used to delay the turbulent laminar transition $[6,7]$. The impact of this control strategy remains however very weak in automobile aerodynamics because the flow is naturally turbulent and the contribution of the friction in the aerodynamic drag is very small (about 10\%).

A simplified car as the Ahmed body [2] is a three-dimensional bluff body moving in the vicinity of the ground generating a turbulent flow. Several separations appear along the body from the front to the back. The resulting recirculation zones contribute to a significant part of the drag coefficient [22], [28]. So, an objective of the control is to reduce the transverse separation area in the wake. The drag coefficient changes strongly with the angle $\alpha$ between the horizontal line and the rear window. For low angles smaller than 12 degrees or large angles higher than 30 degrees, the flow separates at the back and is mainly a two-dimensional base flow. Especially, experimental and numerical studies confirm the two-dimensional behaviour of the detached near-wall flow at the base of the square back Ahmed body geometry [31], [20], [24]. On the contrary for angles between these two values of $\alpha$, the flow is highly three-dimensional over the rear window as there is two counter-rotary lateral vortices and an open separation bulb [28], [20], [31], [25]. In this paper we focus on the square back Ahmed body $\left(\alpha=90^{\circ}\right)$ corresponding to simplified mono space cars or trucks and so a two-dimensional study is performed. The main objective is then to reduce the separated area at the back and thus the static and total pressure gradients that govern the aerodynamic drag.

The use of porous interfaces is a new possibility which can modify the boundary layer behaviour. This passive control technique moves the separation line, reduces the pressure gradients in the near wake and therefore the aerodynamic drag of the vehicles is decreased. A numerical study on the effect of porous layers control on a 2D square cylinder has shown that significant gains on the drag coefficient can be achieved for an appropriate choice of the mechanical parameters (like porous layer permeability and thickness) [10]. In addition a drastic reduction of the vortex induced vibrations on a riser pipe has been obtained [11]. Here the passive control is achieved by means of a porous medium layer which is inserted between the flow and the body. This porous layer modifies the boundary layer effects as it changes the stress forces; indeed the no-slip boundary condition is replaced by a mixed Fourier boundary condition on the walls [13], [1]. The practical difficulty is to compute the flow in two different media, namely the fluid and the porous medium, with different modelling. To overcome this difficulty we use the penalisation method that allows to solve simultaneously both flows with the same model. This method introduced in [4] is studied and validated in [3], applied to turbulent flows in [23] and to flow control in [10]; it consists in adding a penalisation term $U / K$ in the incompressible Navier-Stokes equations 
where $U$ is the velocity vector and $K$ the non dimensional permeability coefficient. It was used as an immersed boundary method to avoid a body fitting and solve directly the flow around bluff bodies on Cartesian meshes. The parameter $K$ is set to a very small value inside the body which corresponds to solve Darcy equations with a very small intrinsic permeability. On the other hand $K$ is set to a very large value in the fluid domain in order to solve only the Navier-Stokes equations as the extra term vanishes. In our application, a third intermediate value of $K$ is used to represent the porous medium where Brinkman equations are solved. In summary we can solve the flow inside the three different media only by changing the value of the parameter $K$ in the penalised Navier-Stokes equations. Besides we avoid to fit the frontiers between the solid body, the porous layer and the fluid.

The goal of this paper is to show how well positioned porous slices can modify the shedding and the near wake, in particular the static pressure gradient to decrease the drag coefficient. In the following we describe the modelling procedure that yields the penalised Navier-Stokes equations or Brinkman-Navier-Stokes equations and give a brief outline of the numerical approximation. Then we give various results of the original flow or the controlled flow around the square back Ahmed body in an open domain or on top of a road. We analyse carefully these results and quantify the efficiency of the passive control using a porous layer.

\section{Modelling and numerical simulation}

In this section we show how an immersed boundary method can be used successfully to model three different media: a solid, a saturated porous medium and an incompressible fluid, with the same equation. In a porous medium the velocity in two dimensions $U=$ $(u, v)$ is given by Darcy law

$$
U=-\frac{k}{\mu \Phi} \nabla p
$$

where $p$ is the pressure, $k$ is the intrinsic permeability, $\mu$ is the viscosity and $\phi$ is the porosity of the fluid. Assuming that the fluid saturating the porous medium is Boussinesq, we get from Brinkman's equation that is valid only for high porosities close to one

$$
\nabla p=-\frac{\mu}{k} \Phi U+\tilde{\mu} \Phi \Delta U
$$

by adding the inertial terms with the Dupuit-Forchheiner relationship [27], the ForchheinerNavier-Stokes equations

$$
\rho \partial_{t} U+\rho(U \cdot \nabla) U+\nabla p=-\frac{\mu}{k} \Phi U+\tilde{\mu} \Phi \Delta U
$$

where $\tilde{\mu}$ is Brinkman's effective viscosity. As $\Phi$ is close to 1 we can approximate $\tilde{\mu} \approx \frac{\mu}{\Phi}$ [33] and get the equation

$$
\rho \partial_{t} U+\rho(U \cdot \nabla) U+\nabla p=-\frac{\mu}{k} \Phi U+\mu \Delta U
$$

Then a non dimensionalisation using the usual substitutions including the mean velocity of the fluid $\bar{U}$ and the height of the domain $H$

$$
\rho=\rho^{\prime} \rho ; U=U^{\prime} \bar{U} ; x=x^{\prime} H ; t=t^{\prime} \frac{H}{\bar{U}} ; p=p^{\prime} \rho|\bar{U}|^{2}
$$


yields the penalised non dimensional Navier-Stokes equations or Brinkman-Navier-Stokes equations for the unknowns $\left(U^{\prime}, p^{\prime}\right)$ denoted $(U, p)$ for the sake of simplicity

$$
\begin{aligned}
\partial_{t} U+(U \cdot \nabla) U-\frac{1}{R e} \Delta U+\frac{U}{K}+\nabla p & =0 & \text { in } \Omega_{T}=\Omega \times(0, T) \\
\operatorname{div} U & =0 & \text { in } \Omega_{T}
\end{aligned}
$$

where $K=\frac{\rho k \Phi \bar{U}}{\mu H}$ is the non dimensional coefficient of permeability of the medium, $R e=$ $\frac{\rho \bar{U} H}{\mu}$ is the Reynolds number based on the height of the body and $\Omega$ is the full domain including the porous layer and the solid body. This model is able to represent the three media as follows. In the fluid the permeability coefficient goes to infinity, the penalisation term vanishes and we solve the non dimensional Navier-Stokes equations. In the solid body the permeability coefficient goes to zero and it has been shown in [3] that solving these equations corresponds to solve Darcy's law in the solid and that the velocity is proportional to $K$. In the porous medium Brinkman equations are recovered. For numerical simulations we set $K=10^{16}$ in the fluid and $K=10^{-8}$ in the solid body; the porous medium is represented by a range of intermediate values varying from $K=10^{-3}$ to $K=100$. Lower values correspond to porous media too close to the solid behaviour to control efficiently the flow. On the opposite, upper values are almost dominated by the flow and thus are equivalent to reduce the size of the obstacle. All the numerical simulations presented in this paper have been performed with $K=10^{-1}$ that appears to be a good intermediate value referring to a parametric study on a wide range of $K$ values [10]. The numerical simulation is performed by solving equations (2.6), (2.7) where $K$ is set at the velocity points according to the medium they belong to. It was shown by different approaches that solving these equations is equivalent to solve the Navier-Stokes equations in the fluid with a Fourier-like boundary condition instead of the no-slip boundary condition (see in particular [8] and [14]). So adding a porous layer between the solid body and the fluid induces a reduction of the shear effects in the boundary layer and thus allows to control the flow. In addition as the pressure is computed inside the solid body, we can compute the drag and lift forces by integrating the penalisation term on the volume of the body [13]:

$$
F_{D}=\int_{b o d y} \frac{u}{K} d x \quad F_{L}=\int_{b o d y} \frac{v}{K} d x
$$

where the body includes the porous layers. To quantify the effect of the control we shall compare the following quantities, the static pressure coefficient $C_{p}$, the drag coefficient $C_{D}$, the root mean-square of the lift coefficient $C_{\text {Lrms }}$ and the enstrophy $Z$ :

$$
\begin{gathered}
C_{p}=2\left(p-p_{0}\right) /\left(\rho|U|^{2}\right) \\
C_{D}=\frac{2 F_{D}}{H} ; \quad C_{L}=\frac{2 F_{L}}{H} \\
C_{L r m s}=\sqrt{\frac{1}{T} \int_{0}^{T} C_{L} d t} Z=\frac{1}{2} \int_{\Omega}|\omega|^{2} d x
\end{gathered}
$$

where $p_{0}$ is the inlet static pressure and $\omega=\partial_{x 1} v-\partial_{x 2} u$ is the scalar vorticity in $2 \mathrm{D}$. Let us note that the static pressure is set to zero at the downstream section of the computational domain. 
The unsteady equations (2.6), (2.7) are associated to an initial datum:

$$
U(x, 0)=U_{0}(x) \text { in } \Omega
$$

and the following boundary conditions:

$$
\begin{gathered}
U=(1,0) \text { on } \Gamma_{D} \\
U=(1,0) \text { on } \Gamma_{0} \\
\sigma(U, p) n+\frac{1}{2}(U \cdot n)^{-}\left(U-U_{r e f}\right)=\sigma\left(U_{r e f}, p_{r e f}\right) n \text { on } \Gamma_{N}
\end{gathered}
$$

where $\sigma$ is the stress tensor and we use the notation $a=a^{+}-a^{-}$. In this paper we consider the flow around the body in an open domain and the flow around the body on top of a road. In the first case the boundary is solely made up of the entrance section $\Gamma_{D}$ and the far field boundary $\Gamma_{N}$ everywhere else. In the second case the road $\Gamma_{0}$ is added as on Figure 1 and the same speed than the flow at infinity is given to the moving ground. The far field boundary condition is used to convey the vortices trough the artificial frontiers without any reflexion (see [9] for more details).

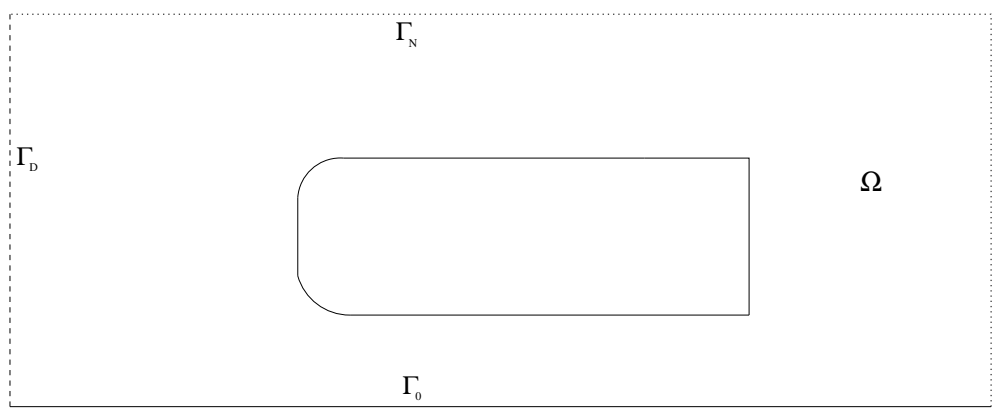

Figure 1: Computational domain including the square back Ahmed body.

The time discretization is achieved by means of the second-order Gear scheme with explicit treatment of the convection term. The space discretization uses a second-order centred scheme for all the linear terms and a third-order upwind scheme for convection terms [12]. The efficiency of the resolution is obtained by a multigrid procedure using a cell-by-cell relaxation smoother.

The numerical tests presented in this paper concern the flow around the Ahmed body without a road or on top of a road. To start we consider the square back Ahmed body. The intermediate porous layers are set on various parts of the body and the corresponding geometries are illustrated on figure 2 and referred as :

case 0 - Ahmed body without porous layer,

case 1 - Ahmed body with a porous layer in the upper circular part in front, case 2 - Ahmed body with a porous layer in the whole top, case 3 - same as case 1 but symmetric, case 4 - same as case 2 but symmetric, case 5 - same as case 4 with the additional front part. 

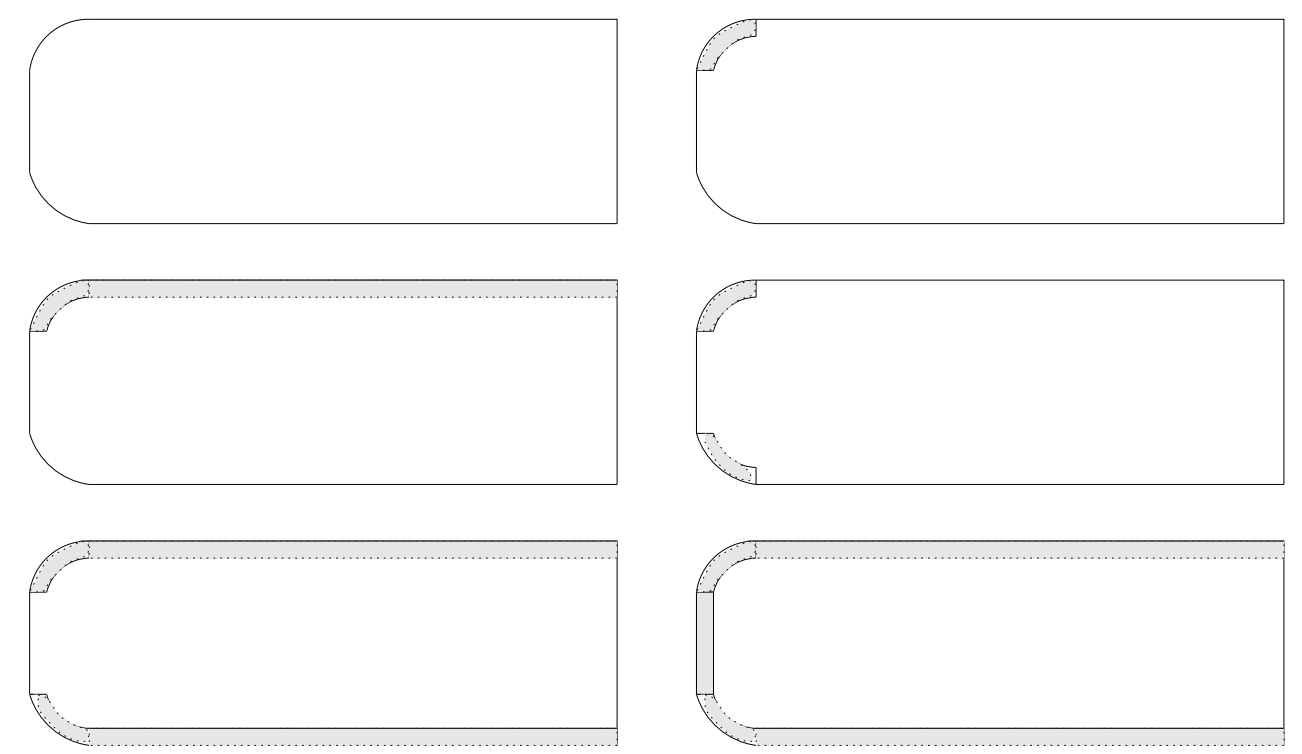

Figure 2: cases 0 (top left), 1 (top right), 2 (middle left), 3 (middle right), 4 (bottom left) and 5 (bottom right) geometries.

The non dimensioned length and height of Ahmed body are respectively $L=3.625$ and $H=1$. The computational domain without a road is $\Omega=(0,12 H) \times(0,7 H)$ and the body is centred in height while the domain is $\Omega=(0,12 H) \times(0,5 H)$ when there is a road and then the body is located at a non dimensioned distance $l=1$ from the road. The results are presented on $1536 \times 896$ and $1536 \times 640$ cells uniform meshes respectively in order to have a significant number of points in the porous layer of thickness $h=0.1 H$. In [10] a complete parametric study on the flow around a square cylinder has shown that the effect of the control is similar for a range of thickness of the porous layer $(0.05 H \leq h \leq 0.2 H)$.

\section{Numerical results and analysis of the passive con- trol}

To comment the numerical results, we plot vorticity fields at given times, the mean flow (vorticity, velocity and pressure fields) and the evolution along time of global quantities such as the enstrophy $Z$, the $\operatorname{drag} C_{D}$ and the root mean-square of the lift coefficient $C_{\text {Lrms }}$ that reveals the regularity and steadiness of the flow. Let us note that up drag refers to the drag due to the front part of the body and down drag refers to the drag at the back. This permits to analyse more carefully the impact of the control. The numerical tests have been performed for the Reynolds number $R e_{r}=R e \times L=30000$ based on the length of the Ahmed body to have a highly transitional flow that corresponds to the studies of the literature [22]. Previous convergence studies have shown that the grid convergence is achieved for such Reynolds numbers on the used grids [11]. The results were then validated, comparing to experimental studies [11]. To have an accurate representation of the solutions we have taken up to 13 points in the porous layers. 


\subsection{Flow around the square back Ahmed body in an open do- main}

As pointed out in the introduction, the near wall flow past the square back Ahmed body is mainly a two-dimensional base flow. So this study is relevant and can describe properly the effect of the porous interfaces on the aerodynamic properties. The interest of the open domain is to have a symmetric geometry on which we can focus on the direct effect of the control procedure only on the body. First of all we see on figure 3 that the unsteady flow is well captured by the numerical method, in particular the alternate vortex shedding at the square back for the non controlled case is close to those observed in [31]. For the other case, it seems that the two porous layers modify the previous shedding generating a diffuse vortical zone in the near wake; we shall discuss the control effect below.

To see the effect of the porous layer we first represent both a zoom of the mean vorticity field and of the mean velocity field around the top circle (figures 4 and 5). The flow velocity inside the porous layers is very low (less than $10 \%$ of the incoming flow velocity) and almost irrotational. For instance in case 2 there is a low speed laminar flow all along the upper porous layer that is expelled at the back in the near wake.

For the case 1 the abrupt discontinuity at the end of the porous layer creates a small vertical jet that increases the shedding. Indeed, the flow inside the porous slice is expelled vertically. This jet increases the transverse size of the wake and thus the drag increases with the pressure drag at the bottom (figures 6 and 7). This physical phenomenon is very well-known in aerodynamics [28]. It increases the asymmetry and the unsteadiness too, so the $C_{L r m s}$ and the enstrophy also increase (table 1). The discontinuity between the porous layer and the solid obstacle induces positive pressure coefficients (the local static pressure is larger than the reference static pressure) as well as higher velocities and the front drag part increases. Both phenomena yield a $22 \%$ total drag increase.
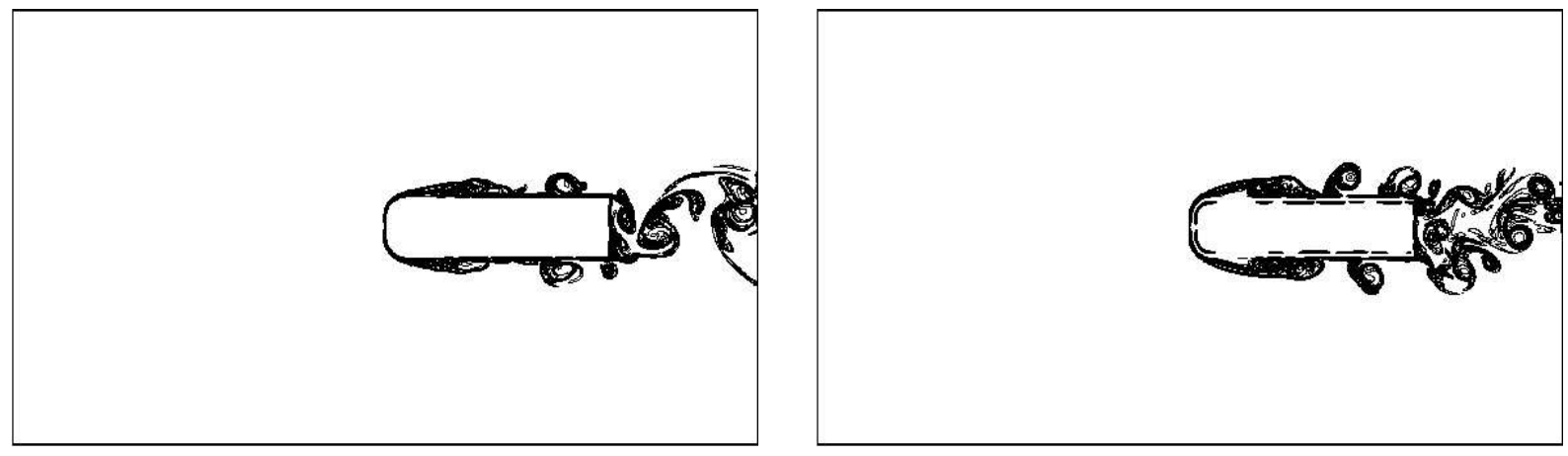

Figure 3: Vorticity field around the square back Ahmed body in an open domain at $R e=30000$. Cases 0 (left) and 5 (right).

To avoid the abrupt discontinuity at the end of the porous layer, we add a whole porous layer on top of the body in case 2 (figure 2). Then the vorticity at the interface between the fluid and the obstacle as well as inside the vortices in the near wake decreases. The flow inside the whole layer has two main consequences: it modifies the shear forces on the top boundary of the body and it induces a low horizontal jet at the back expelled into the wake. Each phenomenon contributes partially to the modification of body forces. It was already shown that the first one is equivalent to change the no-slip boundary con- 

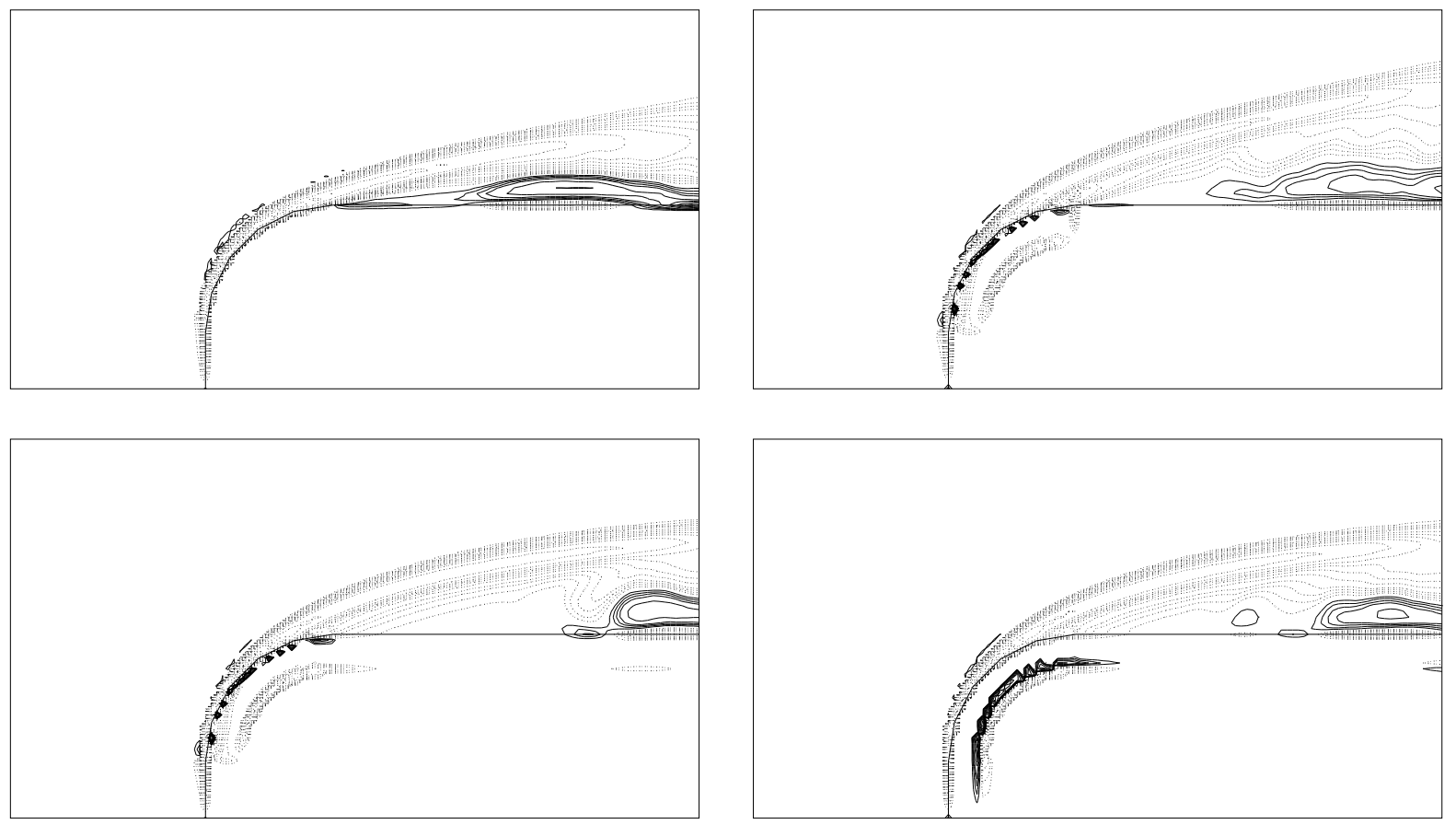

Figure 4: Zooms of the vorticity isolines for the flow around the square back Ahmed body at $R e=30000$. Cases 0 (top left), 1 or 3 (top right), 2 or 4 (bottom left) and 5 (bottom right).
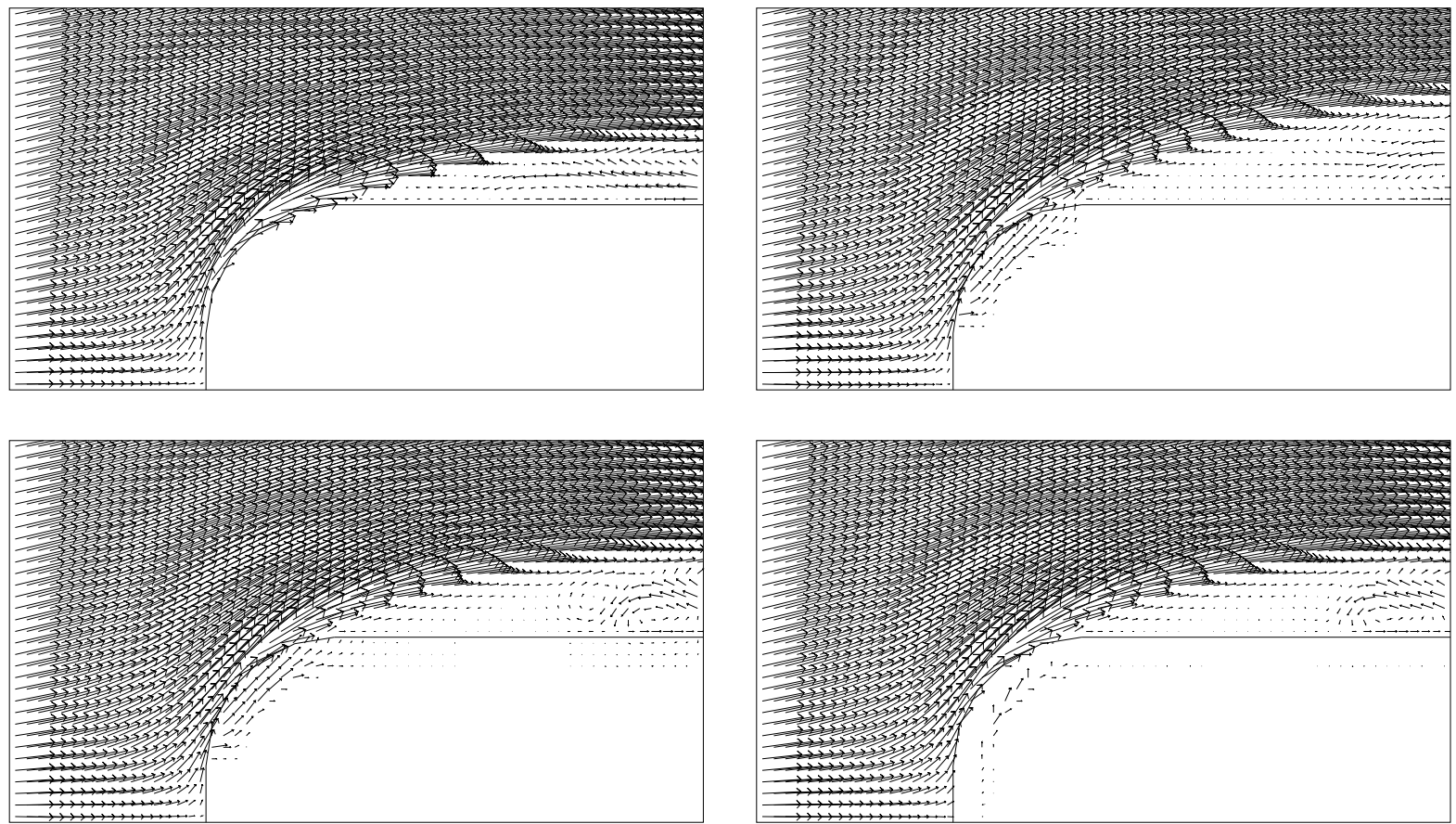

Figure 5: Zooms of the velocity field for the flow around the square back Ahmed body at $R e=30000$. Cases 0 (top left), 1 or 3 (top right), 2 or 4 (bottom left) and 5 (bottom right). 

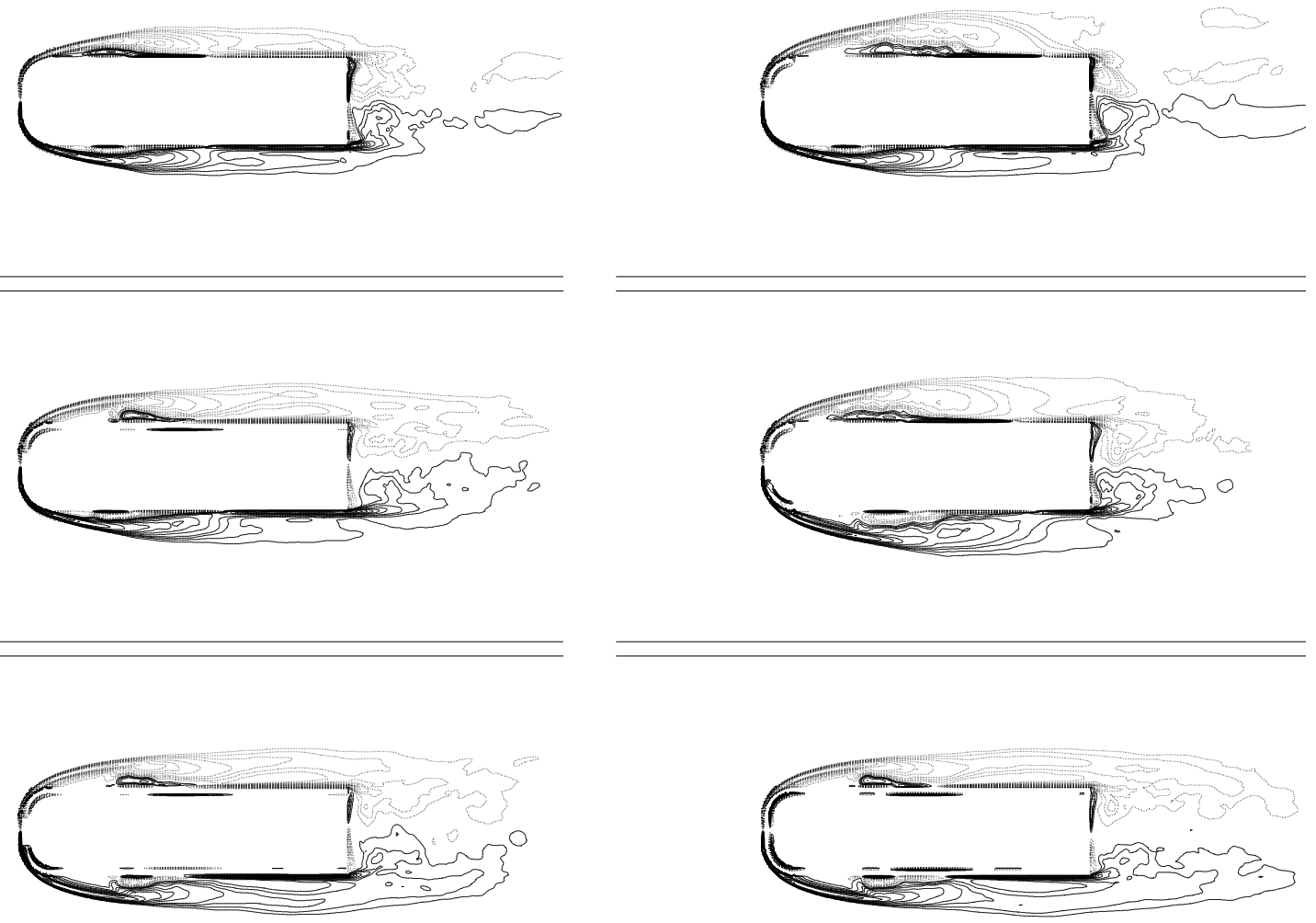

Figure 6: Vorticity isolines for the flow around the square back Ahmed body in an open domain at $R e=30000$. Cases 0 (top left), 1 (top right), 2 (middle left), 3 (middle right), 4 (bottom left) and 5 (bottom right).

\begin{tabular}{||l||c|c|c|c|c||}
\hline & $C_{\text {Lrms }}$ & Enstrophy & Up drag & Down drag & Drag coefficient \\
\hline \hline case 0 & 0.274 & 7911 & 0.133 & 0.329 & 0.469 \\
\hline case 1 & $0.357(+30 \%)$ & 8174 & 0.200 & 0.370 & $0.573(+22 \%)$ \\
\hline case 2 & $0.158(-42 \%)$ & 7877 & 0.130 & 0.120 & $0.257(-45 \%)$ \\
\hline case 3 & $0.254(-7 \%)$ & 8117 & 0.176 & 0.161 & $0.338(-28 \%)$ \\
\hline case 4 & $0.173(-37 \%)$ & 7827 & 0.163 & 0.104 & $0.276(-41 \%)$ \\
\hline case 5 & $0.162(-41 \%)$ & 7876 & 0.185 & 0.088 & $0.281(-40 \%)$ \\
\hline
\end{tabular}

Table 1: Asymptotic values of $C_{\text {Lrms }}$ and mean values of the enstrophy and the drag coefficient for the square back Ahmed body in an open domain at $R e=30000$. 

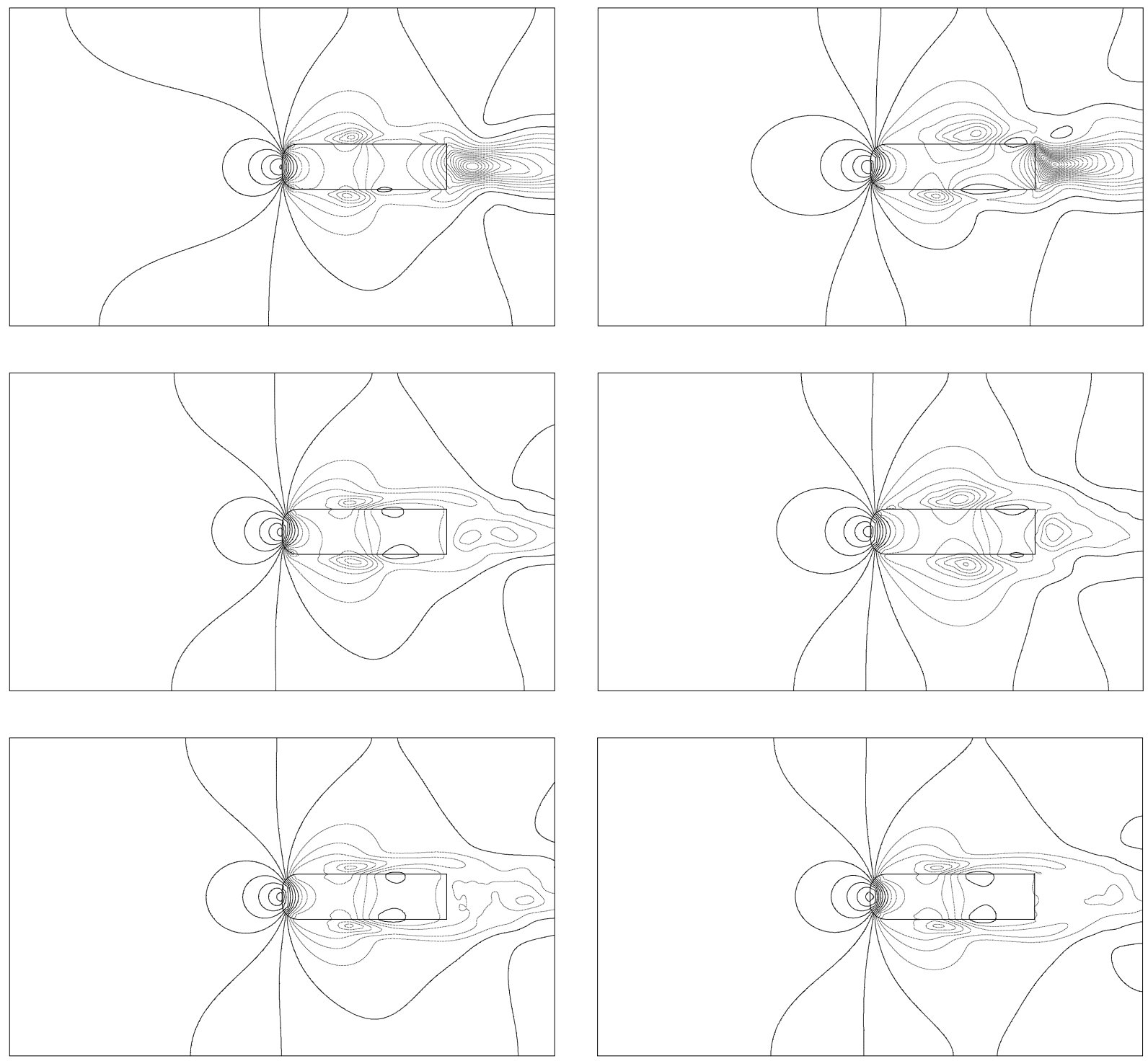

Figure 7: Static pressure coefficient isolines for the flow around the square back Ahmed body in an open domain at $R e=30000$. Cases 0 (top left), 1 (top right), 2 (middle left), 3 (middle right), 4 (bottom left) and 5 (bottom right).

\begin{tabular}{||l||c|c||}
\hline & $P_{\min }$ value in the wake & $P_{\min }$ location \\
\hline \hline case 0 & -1.513 & $(10.20,3.52)$ \\
case 1 & -2.035 & $(10.06,3.55)$ \\
case 2 & -0.464 & $(10.89,3.47)$ \\
case 3 & -0.592 & $(10.02,3.48)$ \\
case 4 & -0.408 & $(11.40,3.42)$ \\
case 5 & -0.311 & $(11.38,3.55)$ \\
\hline
\end{tabular}

Table 2: The value and the location of the non dimensional minimum pressure in the close wake of the square back Ahmed body in an open domain at $\mathrm{Re}=30000$. 

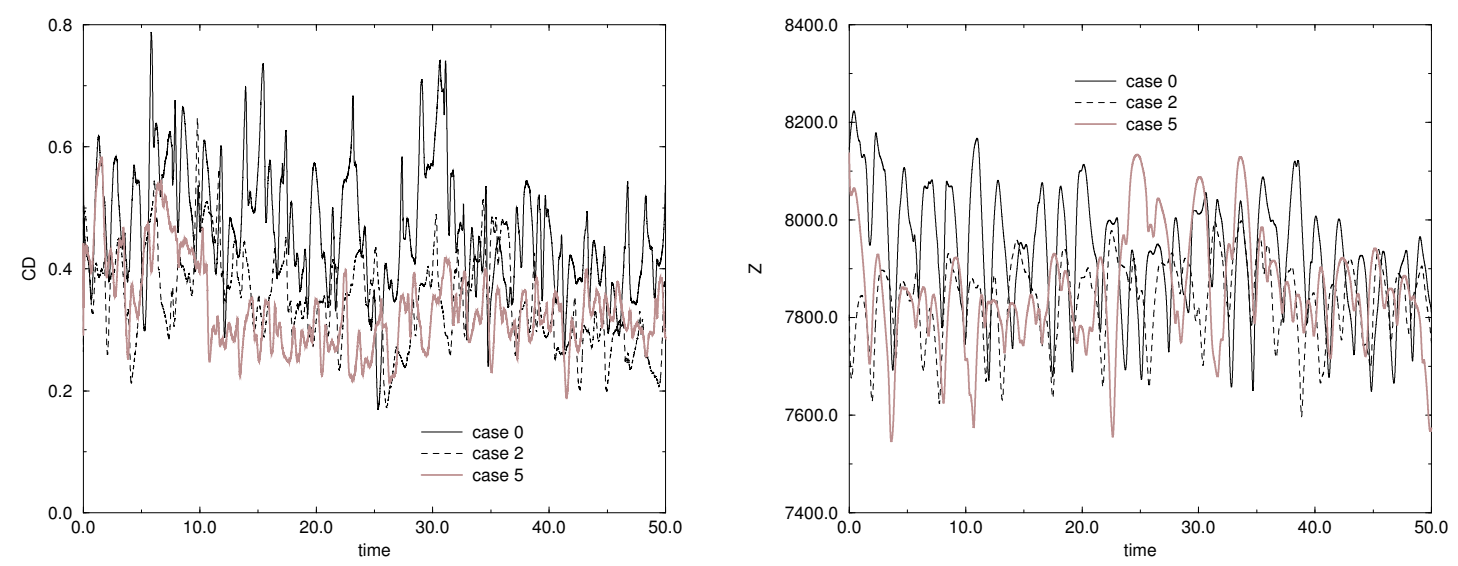

Figure 8: Time history of the drag coefficient (left) and the enstrophy (right) for the flow around the square back Ahmed body in an open domain at $R e=30000$.

dition to a Fourier condition ([1], [14]) and so decreases the boundary layer effects. The influence of the jet at the back is the following: The velocity decreases and the negative mean pressure increases significantly as shown on figure 7 and on table 2. Consequently the two phenomena reduce drastically the bottom drag coefficient. As the contribution of the front part is almost the same than without control (table 1) a $45 \%$ reduction of the total drag coefficient is achieved.

To verify the jet effect of a continuous porous layer on the body end and to quantify it, we performed a new computation to control the flow without porous slices, adding a small uniform equivalent jet on the top back of the body. The results are presented on figure 9 and on table 3; they show that the second phenomenon described above induces a $16 \%$ reduction of the pressure gradient at the back and consequently a $13 \%$ reduction of the drag. This study quantifies approximately the effect of the jet and shows that the modification of the shear forces on the whole top of the body contributes for a large part of the drag reduction.

For the case 3 the same comments than for the case 1 can be made (figure 2); nevertheless we observe very different results with a significant decrease of the drag coefficient. These results suggest that the symmetry might change the vortex shedding mechanism at the base of the Ahmed body and consequently the static pressure is close to zero. The results for the case 4 should be, as we have shown above for case 1 and 3, better than for the case 2. However the results are about the same as for case 2. Indeed, due to the vertical jet, the symmetry is strongly failed in case 1 whereas the case 3 becomes symmetric again. The loss of symmetry changes drastically the alternate vortex shedding at the end of the body. On the contrary in cases 2 and 4 there is no vertical jet and the symmetry is almost conserved; thus the decay is due both to the porous layers and to the small velocities at the end of the body. Finally case 5 gives about the same results than case 4 . All this study shows that, beyond the local action of the passive control by porous layers, there is also a global change linked to the topology of the vortex dynamics that can affect strongly the characteristics of the flow. The numerical experiments above suggest that this global change has a beneficial action in case 3 . The porous layer induces almost a slip property in 
the vicinity of the solid body and consequently reduces the shear forces acting on the body.

Let us make some general comments on the various configurations. The large gradients of the mean vorticity fields correspond to the widest transverse eddies sizes and to the highest drag coefficients. This is in accordance to the results given in [28]. The best results for the aerodynamic drag are achieved when the mean vorticity is close to zero and almost constant at the back. The drag coefficient decreases when the static pressure gradients increase at the back (figure 7 and table 2). This is a classical result for short bluff bodies.

On figure 8 is plotted the time history of the drag and the enstrophy in the cases 0,2 and 5. It is clear that the passive control decreases these quantities as well as their amplitude, their frequency and their irregularities. The table 1 shows that the mean values of these two quantities are significantly reduced (more than 40\%) except in the cases 1 and 3 because of the vertical jet already pointed out. The flow is also regularised as there is also a significant reduction of the $C_{L r m s}$ for the same cases. Consequently the vortex induced vibrations should be strongly reduced. In conclusion it appears that the position of the porous slices is very sensible and that for a good choice a significant improvement can be achieved by this passive control.

All the results obtained at $R e=30000$ are confirmed by simulations with a higher Reynolds number $R e=60000$ as it can be seen on table 4 for cases 2 and 4 . the drag values depend on the Reynolds number but the drag reduction is in the same range. We notice that the results for the symmetric case are this time better.
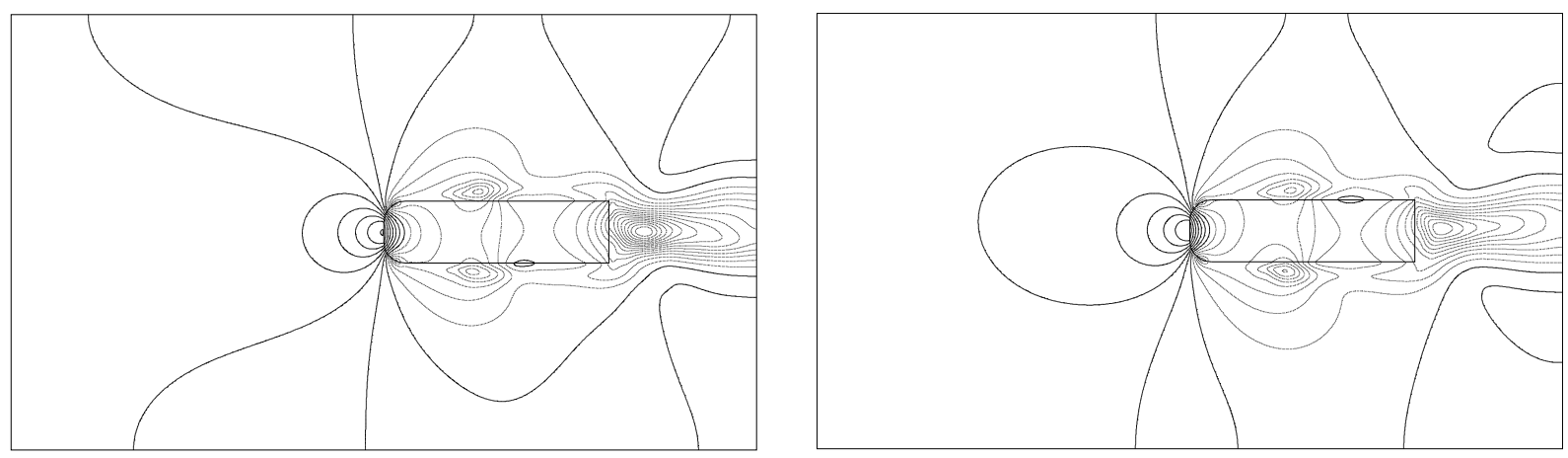

Figure 9: Static pressure coefficient isolines for the flow around the square back Ahmed body in an open domain at $R e=30000$. Case without control (left) and case with control by a permanent jet on top of the back (right).

\begin{tabular}{||l||c|c||}
\hline & Drag coefficient & $P_{\min }$ value in the wake \\
\hline \hline without control & 0.469 & -1.513 \\
with control by a jet & $0.407(-13 \%)$ & $-1.270(-16 \%)$ \\
\hline
\end{tabular}

Table 3: The value of the drag coefficient and of the non dimensional minimum pressure in the close wake of the square back Ahmed body in an open domain at $\operatorname{Re}=30000$. 


\begin{tabular}{||l||c|c|c||}
\hline & $C_{L r m s}$ & Enstrophy & Drag coefficient \\
\hline \hline case 0 & 0.35 & 8268 & 0.549 \\
\hline case 2 & $0.27(-23 \%)$ & 8011 & $0.354(-35 \%)$ \\
\hline case 4 & $0.20(-43 \%)$ & 7931 & $0.310(-44 \%)$ \\
\hline
\end{tabular}

Table 4: Asymptotic values of $C_{L r m s}$ and mean values of the enstrophy and the drag coefficient for the square back Ahmed body in an open domain at $\mathrm{Re}=60000$.

\subsection{Flow around the square back Ahmed body on top of a road}

In this section the same cases as in the previous section are considered except the square back Ahmed body is located on top of a road. As stated before the road is represented by a moving ground having the same speed than the flow at infinity. The results are plotted on figures 10, 11 and 12. The time history of the drag coefficient and the enstrophy are plotted on figure 13 and table 5 summarises the evolution of the three global quantities; The minimum of the mean static pressure in the near wake is given in table 6 . The enstrophy history shows both a strong reduction and a regularisation. The same comments than in the previous section can be done, for instance the vorticity fields plotted on figure 10 show again the negative effect of the small vertical jet in the case 1 and a porous layer on top of the whole roof gives again the best drag reduction. At the back for this last case, the static pressure coefficient gradients are weaker and the vorticity is close to zero (table 6 and figure 11). This is in accordance with the previous results without a road.

So we focus on the effects due to the road. With the road the aerodynamic forces are increased and the flow rate is larger on the upper part of the body. In case 1 the porous layer on top of the circular part of the body has two effects: first the shedding angle is decreased (see [28]); second the flow rate is increased on the upper part of the domain and thus decreased under the car. Consequently the mean velocity under the car is lower and the aerodynamic power dissipated by the floor of the car is decreased [15]. Nevertheless the vertical jet effect described in the open case yields an increase of the up drag. In summary, even if the drag is slightly increased, the loss of the aerodynamic performances is less important than in the open flow case (table 5). The case 2 confirms the previous results and gives again the best results. Here the up drag is decreased as the whole porous slice on top of the body induces a significant increase of the flow rate on top and a decrease of the flow rate under the car. In the other cases the flow rate under the car increases and thus the up drag increases. However, the downstream jet effect under the car is beneficial to the down drag and a global decrease of $30 \%$ is achieved as the mean static pressure coefficient strongly increases in the wake (table 6). In the case 3 the jet effect on the upper part at the bottom of the body is responsible of the increase of the $C_{\text {Lrms }}$. This is mainly due to the oblique jet in the direction to the road that increases locally the pressure forces on the body underneath and thus increases the lift coefficient all along the time.

As in the previous section these results show that the porous layer on the front of the body has not a strong effect on the drag reduction. In the same way the porous layer on the bottom is not very efficient as the gain on the down drag is compensated by a loss on the up drag. The effect of the porous layer is stronger than the effect of the road. As in the previous section the cases 4 and 5 give the lowest static pressure coefficient gradients 
and the case 2 gives the best drag reduction.
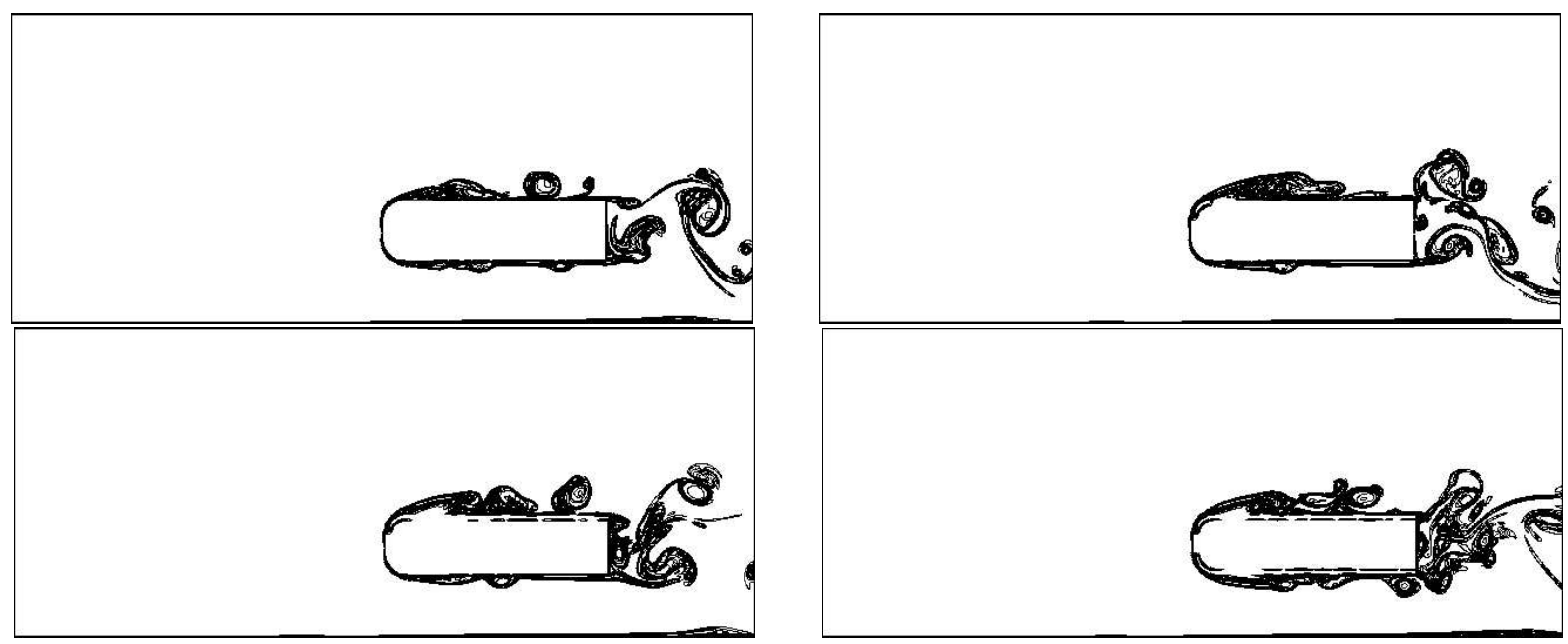

Figure 10: Vorticity field around the square back Ahmed body on top of a road at $\mathrm{Re}=30000$ (top left case 0 , top right case 1 and bottom case 2).

\begin{tabular}{||l||c|c|c|c|c||}
\hline & $C_{\text {Lrms }}$ & Enstrophy & Up drag & Down drag & Drag coefficient \\
\hline \hline case 0 & 0.517 & 827 & 0.173 & 0.343 & 0.526 \\
\hline case 1 & $0.545(+5 \%)$ & $835(+1 \%)$ & 0.231 & 0.330 & $0.567(+8 \%)$ \\
\hline case 2 & $0.396(-23 \%)$ & $592(-28 \%)$ & 0.156 & 0.166 & $0.332(-37 \%)$ \\
\hline case 3 & $0.674(+30 \%)$ & $732(-11 \%)$ & 0.214 & 0.176 & $0.391(-26 \%)$ \\
\hline case 4 & $0.381(-26 \%)$ & $541(-35 \%)$ & 0.213 & 0.139 & $0.362(-31 \%)$ \\
\hline case 5 & $0.352(-32 \%)$ & $533(-36 \%)$ & 0.217 & 0.127 & $0.354(-33 \%)$ \\
\hline
\end{tabular}

Table 5: Asymptotic values of $C_{L r m s}$ and mean values of the enstrophy and the drag coefficient for the square back Ahmed body on top of a road at $\mathrm{Re}=30000$.

\begin{tabular}{||l||c|c||}
\hline & $P_{\min }$ value in the wake & $P_{\min }$ location \\
\hline \hline case 0 & -1.636 & $(10.11,1.53)$ \\
case $\mathbf{1}$ & -1.758 & $(10.11,1.53)$ \\
case 2 & -0.678 & $(10.22,1.39)$ \\
case 3 & -0.850 & $(10.09,1.52)$ \\
case 4 & -0.540 & $(10.89,1.34)$ \\
case 5 & -0.510 & $(10.16,1.34)$ \\
\hline
\end{tabular}

Table 6: The value and the location of the minimum pressure in the close wake of the square back Ahmed body on top of a road at $\mathrm{Re}=30000$. 

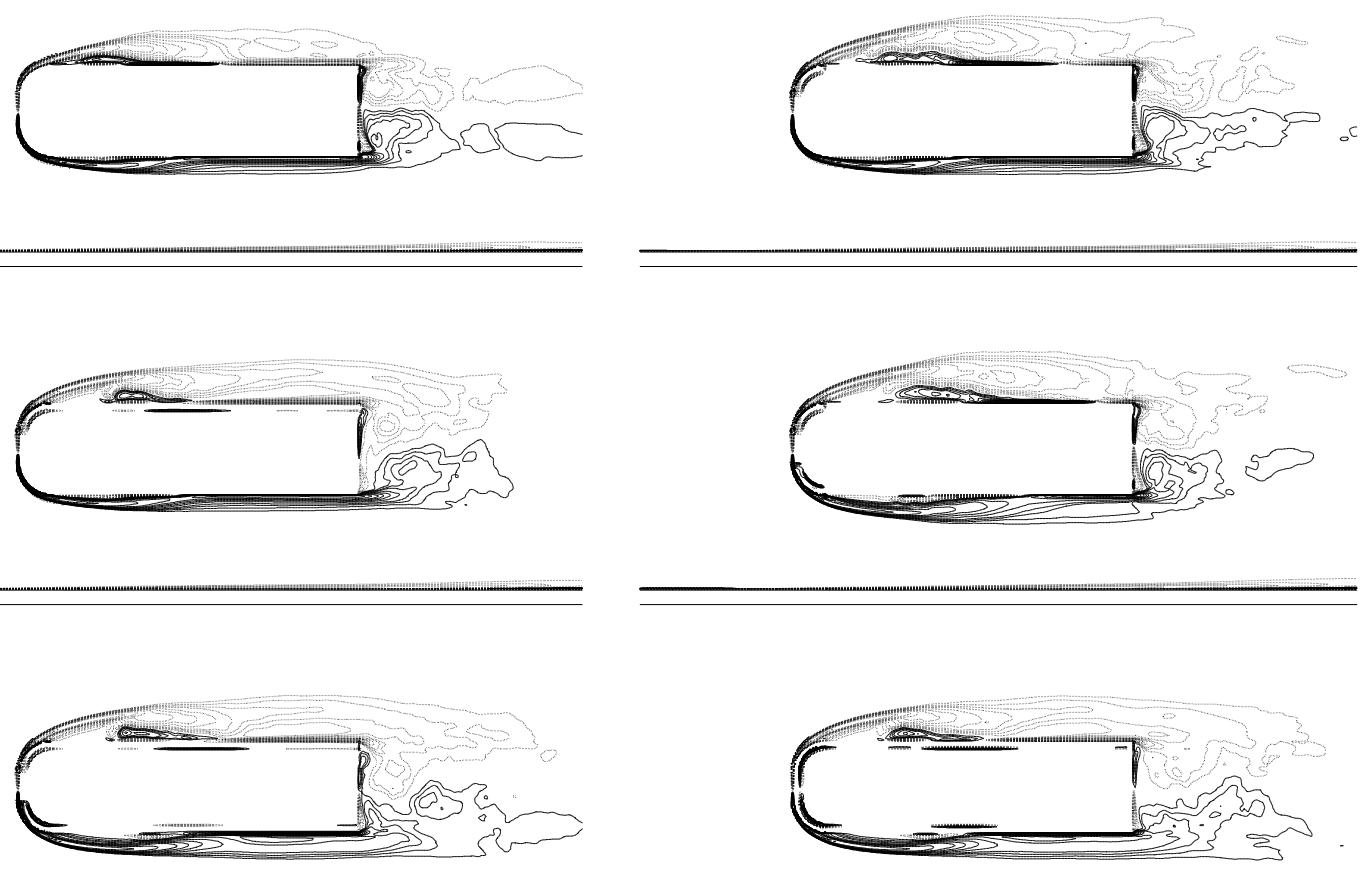

Figure 11: Vorticity isolines for the flow around the square back Ahmed body on top of a road at $R e=30000$. Cases 0 (top left), 1 (top right), 2 (middle left), 3 (middle right), 4 (bottom left) and 5 (bottom right). 

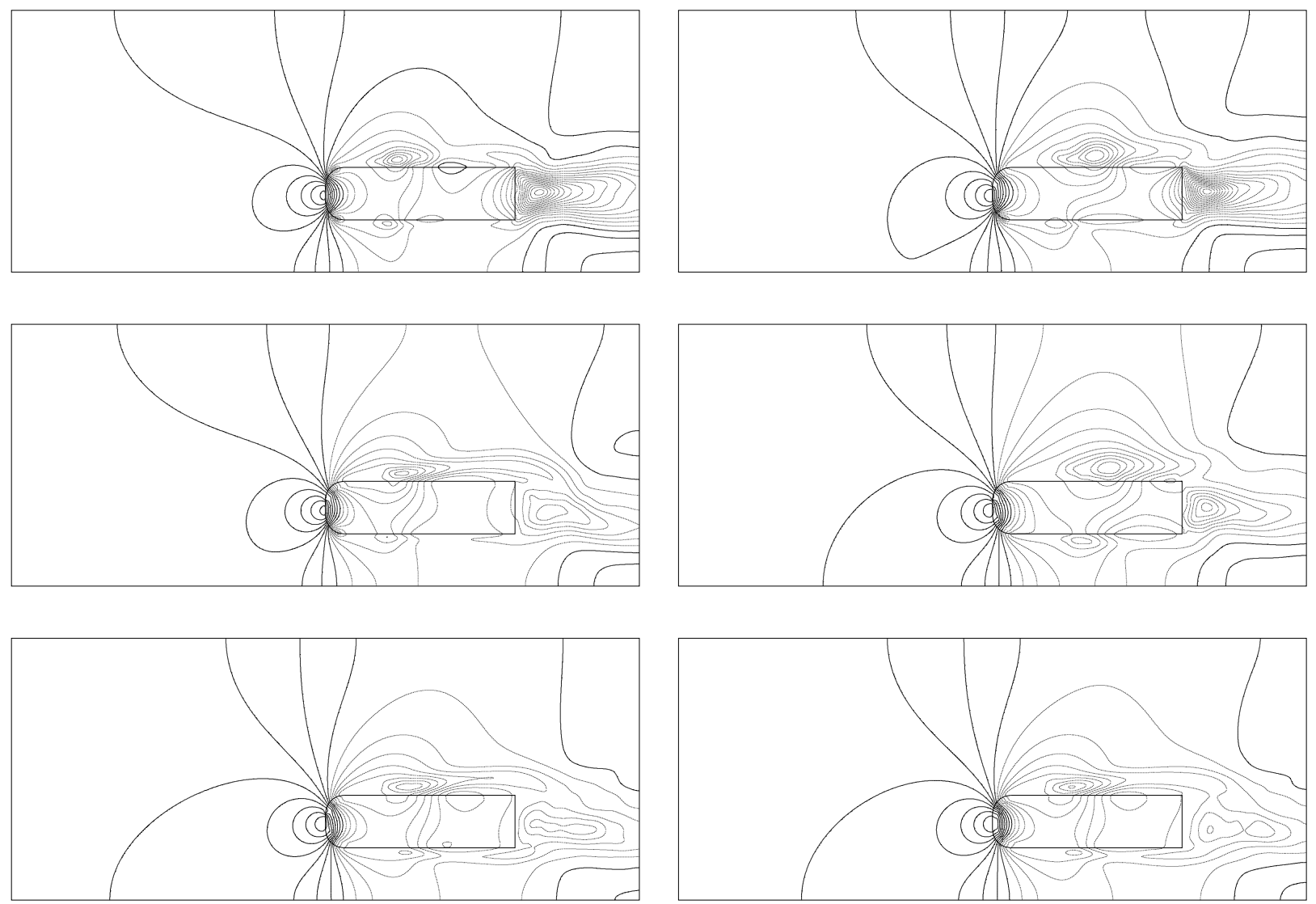

Figure 12: Pressure isolines for the flow around the square back Ahmed body on top of a road at $R e=30000$. Cases 0 (top left), 1 (top right), 2 (middle left), 3 (middle right), 4 (bottom left) and 5 (bottom right).
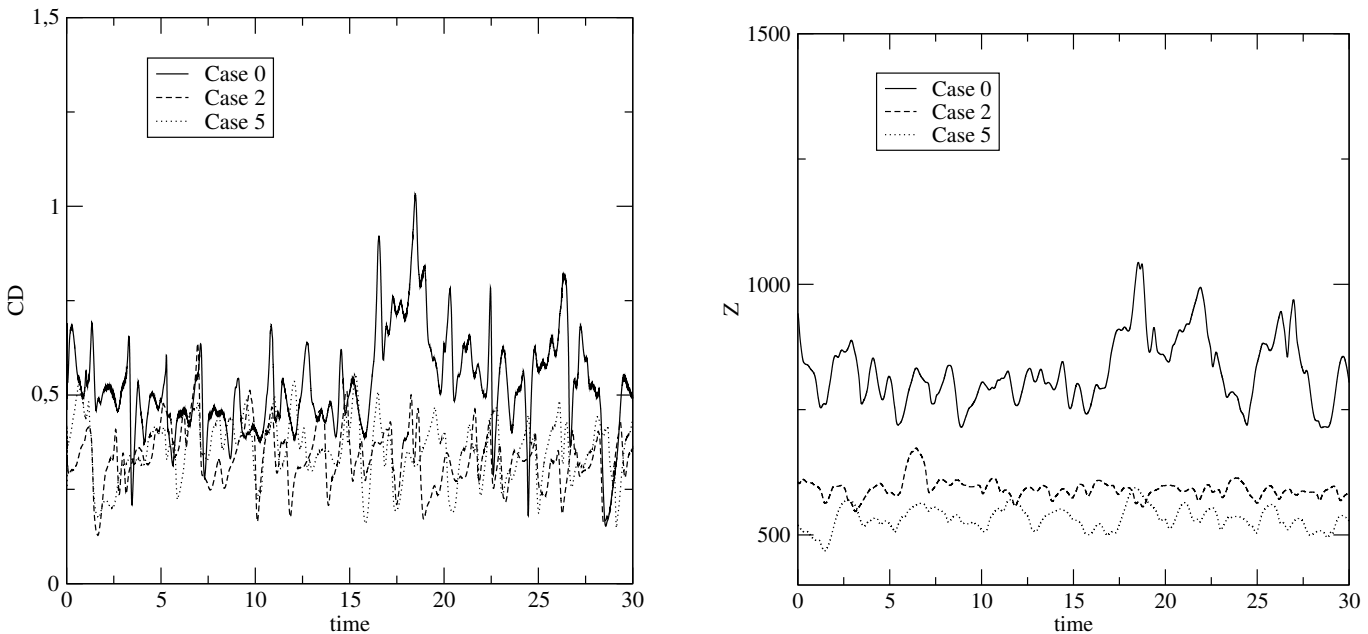

Figure 13: Time history of the drag coefficient (left) and the enstrophy (right) for the flow around the square back Ahmed body on top of a road at $R e=30000$. 


\section{Conclusions}

A passive control technique using a porous slice implemented on some parts of the twodimensional square back Ahmed body is tested to reduce global quantities such as the drag coefficient, the enstrophy or the $C_{\text {Lrms }}$ of the flow. Further, the effect of the road on the control results is studied.

For every successful control configuration, the aerodynamic drag decreases with the rise of the static pressure and the decrease of the vorticity measured at the back. The drag reduction is also linked to the decrease of the wake transverse size. In most cases the results show the beneficial effect of the porous layer implementation on the drag reduction. As an example, a porous layer placed on the upper front corner and roof, reduces considerably the transverse section of the wake and a drag reduction of about $45 \%$ is achieved. The symmetrization of the control devices generally improves the results but adding a porous slice to the vertical front wall does not change much the control. The presence of a road decreases sligthly the efficiency of the control but does not alter the general tendency observed without the road.

In summary this passive control approach is very promising as significant gains are obtained with a good choice of the layers location. Further work on coupling both passive and active control approaches will be performed on the three-dimensional Ahmed body as it is shown in [31] that the flow can be reattached by a suction jet on the top of the rear window.

\section{References}

[1] Y. Achdou, O. Pironneau and F. Valentin, Effective boundary conditions for laminar flows over periodic rough boundaries, J. Comp. Phys. 147, 1998.

[2] S.R. Ahmed, G. Ramm and G. Falting, Some salient features of the time averaged ground vehicle wake, SAE paper 840300, 1984.

[3] Ph. Angot, Ch.-H. Bruneau and P. Fabrie, A penalization method to take into account obstacles in incompressible viscous flows, Numer Math 81, 1999.

[4] E. Arquis and J. P. Caltagirone, Sur les conditions hydrodynamiques au voisinage d'une interface milieu fluide - milieu poreux : application à la convection naturelle, C. R. Acad. Sci. Paris, Série II 299, 1984.

[5] C. Bak, P. Fuglsang, J. Johansen and I. Antoniou, Wind tunnel tests of the NACA 63-415 and modified NACA 63-415 airfoil, Riso-R-1193 Riso National Laboratory 2000.

[6] P. W. Bearman, Investigation of the flow behind a two-dimensional model with a blunt trailing edge with splitter plates, J. Fluid Mech. 21, 1965.

[7] P.W. Bearman and J.K. Harvey, Control of circular cylinder flow by the use of dimples, AIAA J. 31, 1993.

[8] G.D. Beavers and D.D. Joseph, Boundary conditions at a naturally permeable wall, J. Fluid Mech. 30, 1967.

[9] C.-H. Bruneau and P. Fabrie, Effective downstream boundary conditions for incompressible Navier-Stokes equations, Int. J. Num. Meth. Fluids 19, 1994. 
[10] C.-H. Bruneau and I. Mortazavi, Passive control of flow around a square cylinder using porous media, Int. J. Num. Meth. Fluids 46, 2004.

[11] Ch.-H. Bruneau and I. Mortazavi, Control of vortex shedding around a pipe section using a porous sheath, Int. J. Offshore and Polar Engg $16 n^{0}$ 2, 2006.

[12] C.-H. Bruneau and M. Saad, The 2d lid-driven cavity problem revisited, Computers Es Fluids $35 n^{0}$ 3, 2006.

[13] J.-P. Caltagirone, Sur l'interaction fluide-milieu poreux : Application au calcul des efforts exercés sur un obstacle par un fluide visqueux, CRAS 318 série II, 1994.

[14] G. Carbou, Asymptotic analysis for a two-scales penalization method in fluid dynamics, $J$ Math Fluid Mech, in print.

[15] L. Chometon and P. Gillieron, Modélisation des écoulements tridimensionnels décollés autour des véhicules automobiles à l'aide d'un modèle à zéro-dimension, Journée d'étude aérodynamique automobile, Courbevois-Paris 5 850288, 1996.

[16] K.R. Cooper, The effect of front-edge rounding and rear-edge shaping on the aerodynamic drag of bluff vehicles in ground proximity, SAE Paper 850288, 1985.

[17] H.E.Fiedler and H.H.Fernholz, On management and control of turbulent shear flows, Program Aerospace Sci. 27, 1990.

[18] P. Gilliéron, Contrôle des écoulements appliqué à l'automobile, état de l'art, Mécanique et Industrie Elsevier, 2002.

[19] P. Gilliéron, Detailed analysis of the overtaking process, J. Mech. Eng 53, 2003.

[20] P. Gilliéron and F. Chometon, Modelling of stationary three-dimensional detached airflows around an Ahmed Reference Body, ESAIM Proc 7, 1999.

[21] P. Gilliéron, F. Chometon and J. Laurent, Analysis of histeresis and phase shifting phenomena in unsteady three-dimensional wakes, Experiments in Fluids 35, 2003.

[22] P. Gilliéron and A. Spohn, Flow separations generated by a simplified geometry of an automotive vehicle, IUTAM Symp. Unsteady Separated Flows 2002.

[23] N. Kevlahan and JM Ghidaglia, Computation of turbulent flow past an array of cylinders using a spectral method with Brinkman penalization, Eur J Mech B Fluids 20, 2001.

[24] S. Krajnovic and L. Davidson, Numerical study of the flow around the Bus-Shaped body, ASME Journ. Fluid Engineering 125, 2003.

[25] H. Lienhart, C. Stoots and S. Becker, Flow and turbulence structures in the wake of a simplified car model (Ahmed model), DGLR Fach Symp. Der AG STAB 2000.

[26] W.A. Mair, The effect of a rear-mounted disc on the drag of a blunt-based body of revolution, Aeronautical Quarterly 16, 1965.

[27] D.A. Nield, A. Bejan, Convection in porous media, Springer, 1999.

[28] M. Onorato, A. Costelli and A. Garonne, Measurement through wake analysis, SAE Paper $\mathbf{5 6 9}, 1984$. 
[29] R. Rezncek, Surface tracing methods, chapter 7, Handbook of Flow Visualization Edited by Wen Jei-Yang, Hemisphere Publishing, 1989.

[30] A. Roshko and K. Koening, Interaction effects on the drag of bluff bodies in tandem, Symposium on Aerodynamic Drag Mechanisms of Bluff Bodies and Road Vehicles, General Motors Report 1976.

[31] M. Rouméas, Contribution à l'analyse et au contrôle des sillages de corps épais par aspiration ou soufflage continu, $\mathrm{PhD}$ thesis Toulouse, 2006.

[32] Y. Suzuki and T. Ijima, Profile drag of circular cylinders with surface roughness of straight knurls, Flucome 94, Toulouse 1994.

[33] S. Whitaker, The method of volume averaging, Kluver, 1999. 